\title{
Fractional dynamics in liquid manipulation
}

\author{
M.F.M. LIMA ${ }^{1 *}$ and J.A.T. MACHADO ${ }^{2}$ \\ ${ }^{1}$ Dept. of Electrical Engineering, Superior School of Technology, Polytechnic Institute of Viseu, 3504-510 Viseu, Portugal \\ ${ }^{2}$ Dept. of Electrical Engineering, Institute of Engineering, Polytechnic Institute of Porto, 4200-072 Porto, Portugal
}

\begin{abstract}
This paper presents a fractional calculus perspective in the study of signals captured during the movement of a mechanical manipulator carrying a liquid container. In order to study the signals an experimental setup is implemented. The system acquires data from the sensors, in real time, and, in a second phase, processes them through an analysis package. The analysis package runs off-line and handles the recorded data. The results show that the Fourier spectrum of several signals presents a fractional behavior. The experimental study provides useful information that can assist in the design of a control system and the trajectory planning to be used in reducing or eliminating the effect of vibrations.
\end{abstract}

Key words: fractional order system, vibrations, robotics, sensors, Fourier transform.

\section{Introduction}

In practice the robotic manipulators present some degree of unwanted vibrations. In fact, the advent of lightweight arm manipulators, mainly in the aerospace industry, where weight is an important issue, leads to the problem of intense vibrations. On the other hand, robots interacting with the environment often generate impacts that propagate through the mechanical structure and produce also vibrations.

Motivated by the problem of vibrations, this paper studies the robotic signals captured during the motion of a spherical container attached to the manipulator. The container carries a liquid and its acceleration induces motion of the content causing consequently a liquid vibration. The study is done in a fractional calculus (FC) perspective. In order to analyze the phenomena involved an acquisition system was developed. The manipulator motion produces vibrations, either from the structural modes or from the liquid vibration. The instrumentation system acquires signals from multiple sensors that capture the axis positions, mass accelerations, forces and moments and electrical currents in the motors. Afterwards, the analysis package, running off-line, reads the data recorded by the acquisition system and examines them.

Bearing these ideas in mind, this paper is organized as follows. Section 2 addresses the motivation for this work. Section 3 describes the robotic system enhanced with the instrumentation setup. Section 4 presents the experimental results. Finally, Sec. 5 draws the main conclusions and points out future work.

\section{Motivation}

Reference [1] mentions several techniques for reducing vibrations and its implementation either at the robot manufacturing stage or at the operational stage. Briefly, the techniques can be enumerate as: $(i)$ conventional compensation, (ii) structural damping or passive vibration absorption, (iii) control based on the direct measurement of the absolute position of the gripper, $(i v)$ control schemes using the direct measurement of the modal response, $(v)$ control driving, actively, energy out of the vibration modes, (vi) use a micromanipulator at the endpoint of the larger manipulator and (vii) adjustment of the manipulator command inputs so that vibrations are reduced or eliminated.

In recent years the study of micro/macro robotic manipulators has been receiving considerable attention. This approach was employed in manipulators that have been proposed for space applications and nuclear waste cleanup. Several authors have studied this technique [2], namely [3] and [4] that adopted the command filtering approach in order to position the micromanipulator. Also, [4] and [5] used inertial damping techniques taking advantage of a micro manipulator located at the end of a flexible link. In this perspective, to control the macro/micro system, in order to eliminate or reduce the effect of the vibration, is fundamental to study the involved variables.

One of the applications where the vibration occurs is in the manipulation of liquids. Several authors addressed the dynamics problem due to liquid slosh loads. There are several mathematical tools to describe the fluids. For example, NavierStokes equations $[6,7]$ can be used to model the liquid dynamics. Concerning the problem of control the liquid vibration, it was first encountered in control of guided missiles in the aerospace industry. In this application it was found that sloshing in the fuel tanks could result in instabilities. Lately, movement of open containers containing fluid, e.g. molten metal and various beverages, has been investigated. The main goal is then to move the container as fast as possible without too much slosh $[7,8]$.

Bearing these ideas in mind, this article studies the robotic signals, in a FC perspective. In fact, the study of fractional order systems has been receiving considerable attention $[9,10]$

*e-mail: lima@mail.estv.ipv.pt 
due to the facts that many physical systems are well characterized by fractional models [11]. With the success in the synthesis of real noninteger differentiators, the emergence of new electrical elements [12, 13], and the design of controllers [14], fractional algorithms have been applied in a variety of dynamical processes [15]. Therefore, the study presented here can assist in the design of the control system to be used.

\section{Experimental platform}

The developed experimental platform has two main parts: the hardware and the software components [16, 17]. The hardware architecture is shown in Fig. 1 and it is made up of a robot manipulator, a Personal Computer and an interface electronic system.

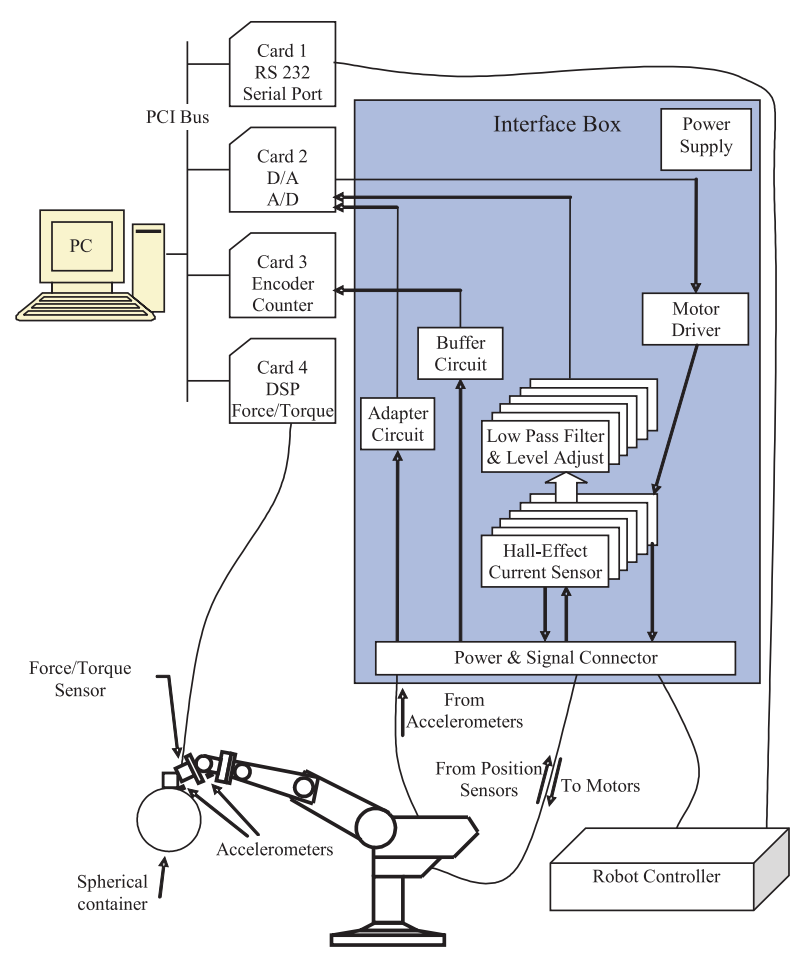

Fig. 1. Block diagram of hardware architecture

The interface box is inserted between the robot arm and the robot controller, not only to acquire the internal robot signals; but also to capture external signals, such as those arising from accelerometers and force/torque sensors. The modules are made up of electronic cards specifically designed for this work. The function of the modules is to adapt the signals and to isolate galvanically the robot's electronic equipment from the rest of the hardware required by the experiments. The software package runs in a Pentium 4, 3.0 GHz PC and consists of two applications: the acquisition application and the analysis package.

The acquisition application is a real time program for acquiring and recording the robot signals.

After the real time acquisition, the analysis package processes the data off-line in two phases, namely, preprocessing and processing. The preprocessing phase consists on the signal selection in time, and their synchronization and truncation. The processing stage implements several algorithms for signal processing such as the auto and cross correlation, Fourier transform (FT), and window Fourier transform.

\section{Experimental results}

In the experiment a spherical container is adopted. Its physical properties are shown in Table 1. To test the behavior of the variables in different situations, the container can remain empty or its content can be a liquid or a solid. Figure 2 depicts the robot with the container. The robot motion is programmed in a way that the container moves from an initial to a final position following a linear trajectory. The distance between the points is $0.6 \mathrm{~m}$.

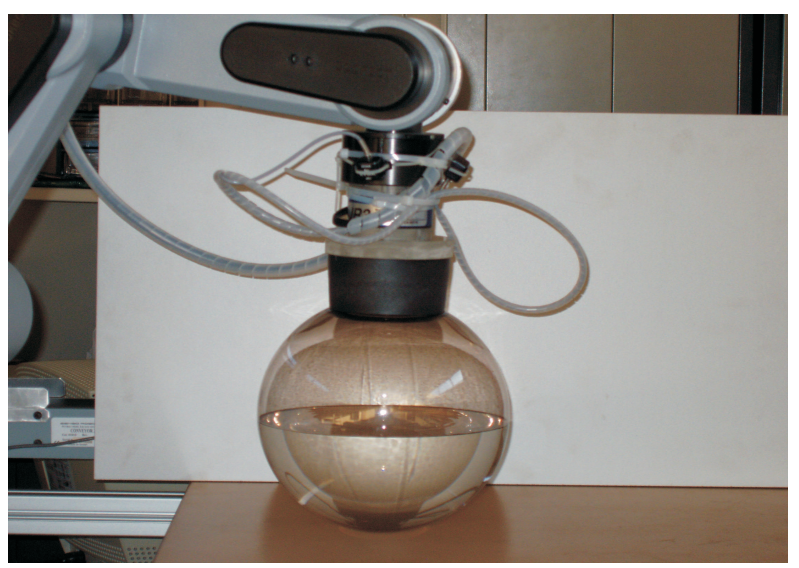

Fig. 2. Spherical container with liquid

Table 1

Physical properties of the spherical container

\begin{tabular}{cc}
\hline \hline Characteristic & Spherical container \\
\hline Mass (empty) $[\mathrm{kg}]$ & $215 \times 10^{\mathrm{tz}}$ \\
\hline Diameter $[\mathrm{m}]$ & $203 \times 10^{\mathrm{tz}}$ \\
\hline
\end{tabular}

During the motion of the manipulator the container is moved by the robot and several signals are recorded with a sampling frequency of $f_{s}=500 \mathrm{~Hz}$. The signals come from different sensors, such as accelerometers, force and torque sensor, position encoders and current sensors. The signals are captured for three different situations: $(i)$ empty container, (ii) container with a solid, and (iii) container with a liquid. The container with the solid or the liquid have an identical mass, namely of $1 \mathrm{~kg}$. In the experiment the used liquid is water. The acceleration of the container induces motion of the liquid. This is referred to as slosh or liquid vibration. The amount of slosh depends on how the container is accelerated, the geometry of the container and the properties of the fluid.

Time domain. The time evolution of some variables is shown in the Figs. 3-6 corresponding to the cases: (i) empty container, (ii) container with a solid, and (iii) container with a liquid.

To analyze the vibration effect of the liquid, caused by the container acceleration, the signals are captured during $20 \mathrm{~s}$, although the motion of the container is executed in approximately $5 \mathrm{~s}$. 
In order to test different acceleration shapes two types of trajectory velocity are used: the trapezoidal and the parabolic profiles. The trapezoidal profile causes the motors to accelerate and decelerate quickly at the start and end of movement, with a constant speed along the path. The parabolic profile causes the motors to accelerate slowly until maximum speed is reached, then decelerate at the same rate.

Figure 3 represents the electrical current of the motors for the trapezoidal trajectory velocity profile. As consequence the robot joints rotate which causes the container motion. Figures 4 shows the moments caused by the container motion. The effect of the liquid vibration can be observed in the $M_{y}$ moment component.
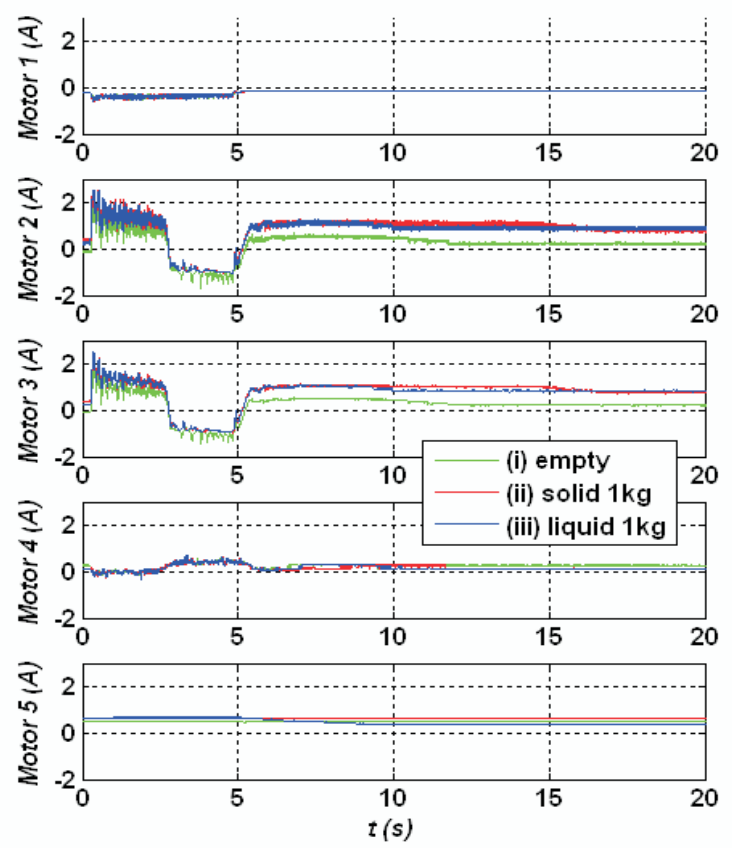

Fig. 3. Electrical currents of robot axis motors for the trapezoidal profile
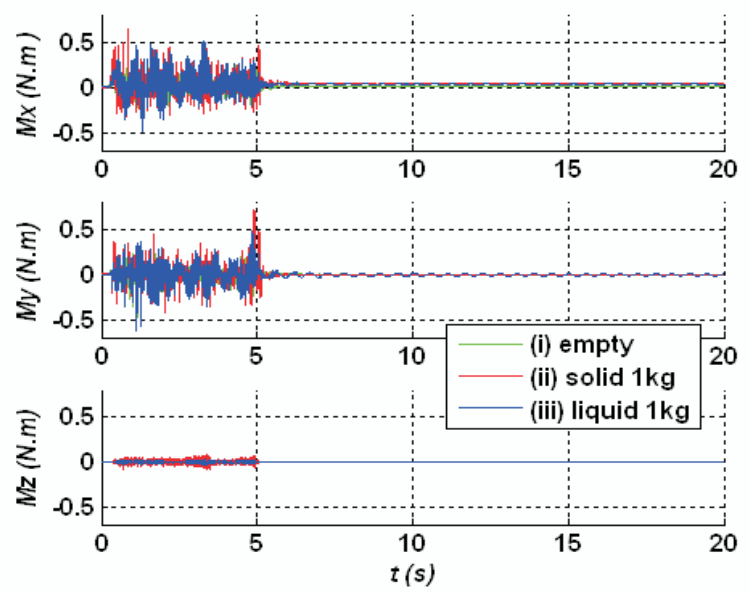

Fig. 4. Moments at the gripper sensor for the trapezoidal profile

Figures 5-6 show the time evolution of the variables for the parabolic trajectory velocity profile. The dynamics of the signal positions at the start and end of movement are smoother for the parabolic case. This fact is reflected in the electrical currents of the robot axis motors (Figs. 3 and 5).
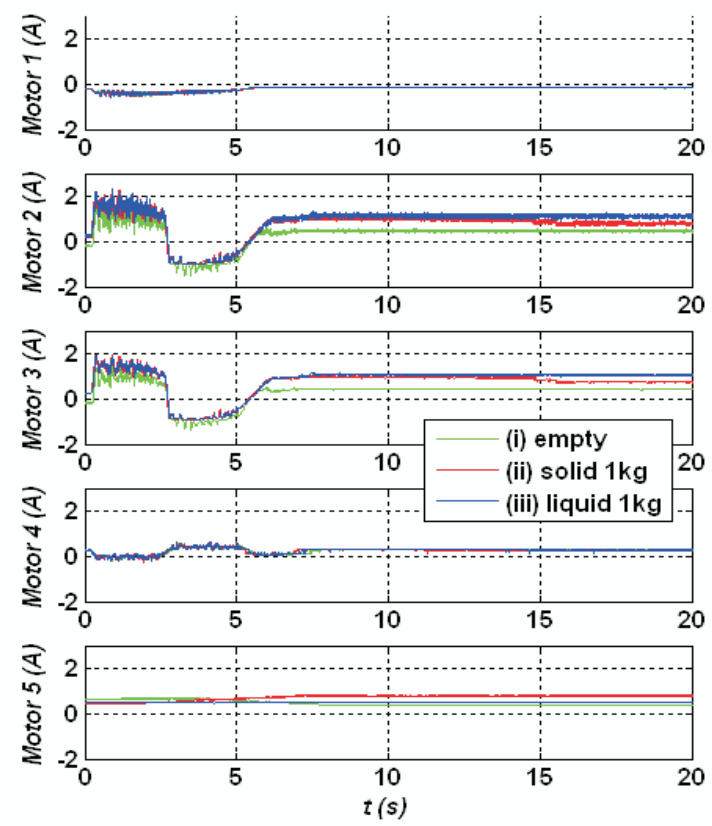

Fig. 5. Electrical currents of robot axis motors for the parabolic profile
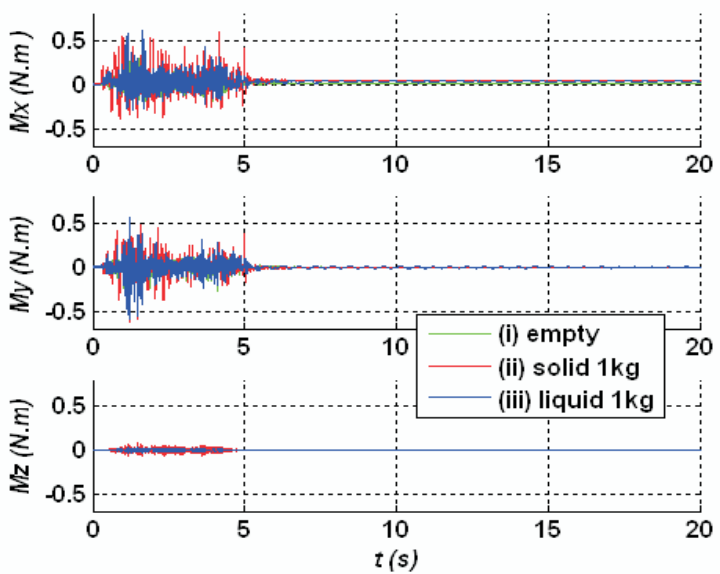

Fig. 6. Moments at the gripper sensor for the parabolic profile

The smoother dynamics of the parabolic profile has a consequence of lower forces induced in the container. Therefore, the amplitude of the liquid vibration, caused by the movement of the container, is lower than the acceleration occurring in the trapezoidal case. This fact is reflected in the moments measured at the gripper sensor (see the zoom in Figs. 4 and 6).

Frequency domain. In order to examine the behavior of the signal FT a trendline is superimposed within the spectrum over, at least, one decade. the trendline is based on a power law approximation [18]:

$$
|F\{f(t)\}| \approx c \omega^{m}
$$


where $F$ is the Fourier operator, $c \in \Re$ is a constant that depends on the amplitude, $\omega$ is the frequency and $m \in \Re$ is the slope.

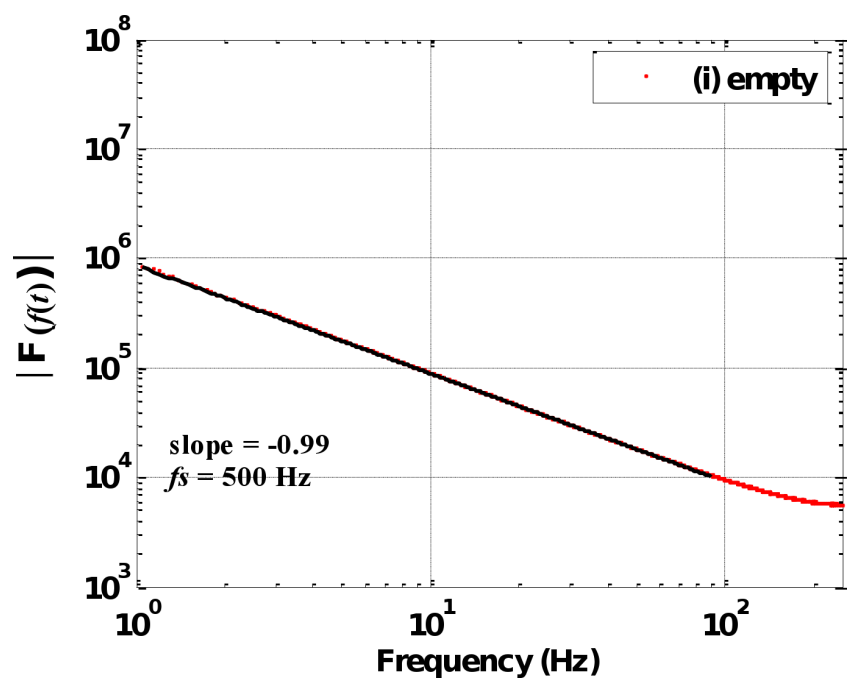

Fig. 7. Spectrum of the axis 1 position for the trapezoidal profile
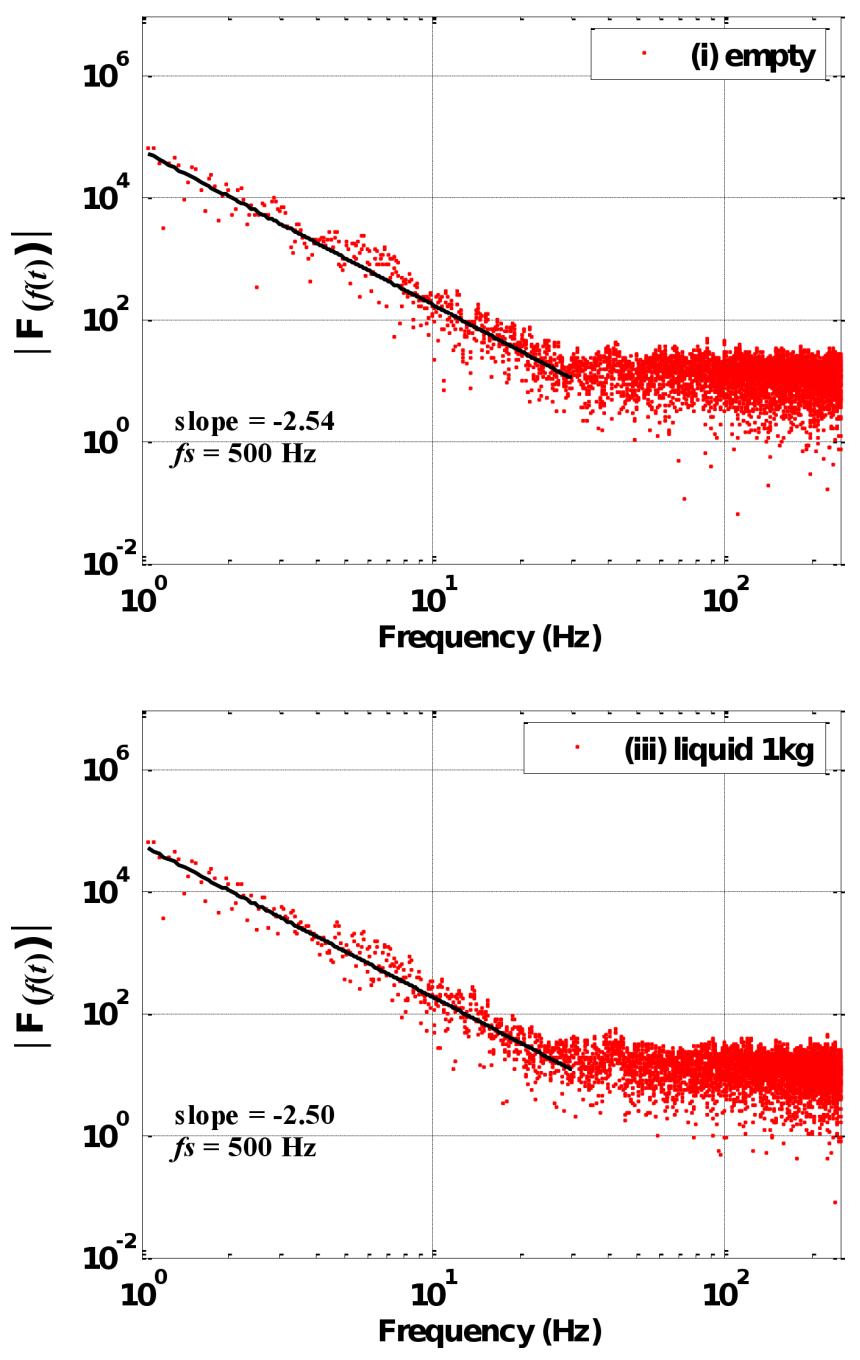

Fig. 8. Spectrum of the axis 3 position for the trapezoidal profile
All the signals of the trajectories captured by the acquisition system were studied but, only the most relevant are depicted.

Figure 7 shows the amplitude of the Fast Fourier Transform (FFT) of the axis 1 position signal (case $i$ ). A trendline with slope $m=-0.99$ was calculated revealling, clearly, the integer order behavior. The position signals present identical characteristics, in terms of its spectrum, for the others cases (ii) container with a solid and (iii) container with a liquid. In fact the position signals maintain the same shape for the three cases [19].

Figure 8 shows the amplitude of the FFT of the axis 3 position signal (case $i$ and case iii). The spectrum amplitude is also approximated by trendlines in a frequency range larger than one decade. The trendlines present slopes that vary slightly (slope $m=-2.54$ for case $i$ and slope $m=-2.50$ for case iii). The study of the case $i$ ) presents a trendline with a slope of $m=-2.62$. Therefore, the lines reveal, clearly, fractional order behavior in all cases.

The others position signals (axis 2 and 4) were studied, revealing also a well defined spectrum. Their trendlines present middle slope values that are difficult to classify in terms of its behavior as fractional or integer order. In what concerns to the axis 5 signal position signal, as it maintains the same value during all time acquisition, it consists only in a direct current (DC) component.

Figure 9 shows, as an example, the FFT amplitude of the electrical current for the motor axis 3 , that occurs in the case of the trapezoidal profile with container carrying a liquid (case iii). A trendline with slope $m=-1.19$ is calculated in a frequency range larger than one decade and superimposed over the spectrum. The others currents were studied, revealing also an identical behavior in terms of its spectrum spread, for the tested conditions (cases $i, i i$ and $i i i)$.

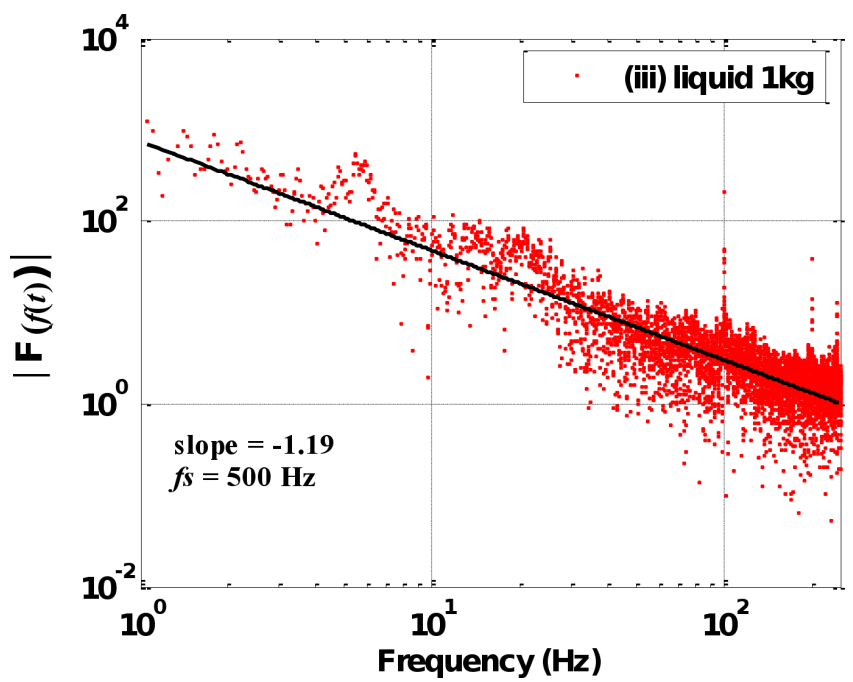

Fig. 9. Spectrum of the axis 3 motor current for the trapezoidal profile

According to the robot manufacturer specifications [20] the loop control of the robot has a cycle time of $t_{c}=10 \mathrm{~ms}$. This fact is observed approximately at the fundamental $\left(f_{c}=\right.$ 
$100 \mathrm{~Hz}$ ) and multiple harmonics in all spectra of motor currents (Fig. 9).

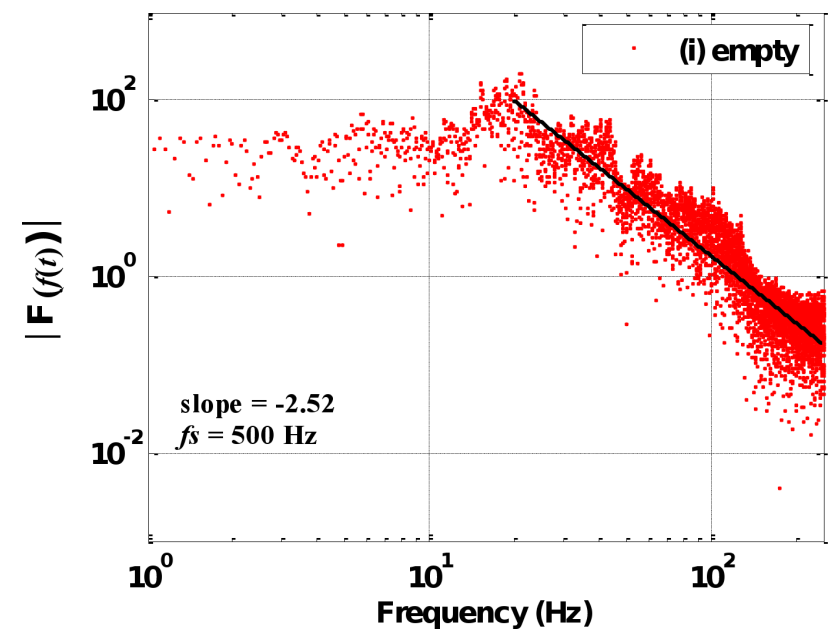

Fig. 10. Spectrum of the $F_{x}$ force for the trapezoidal profile
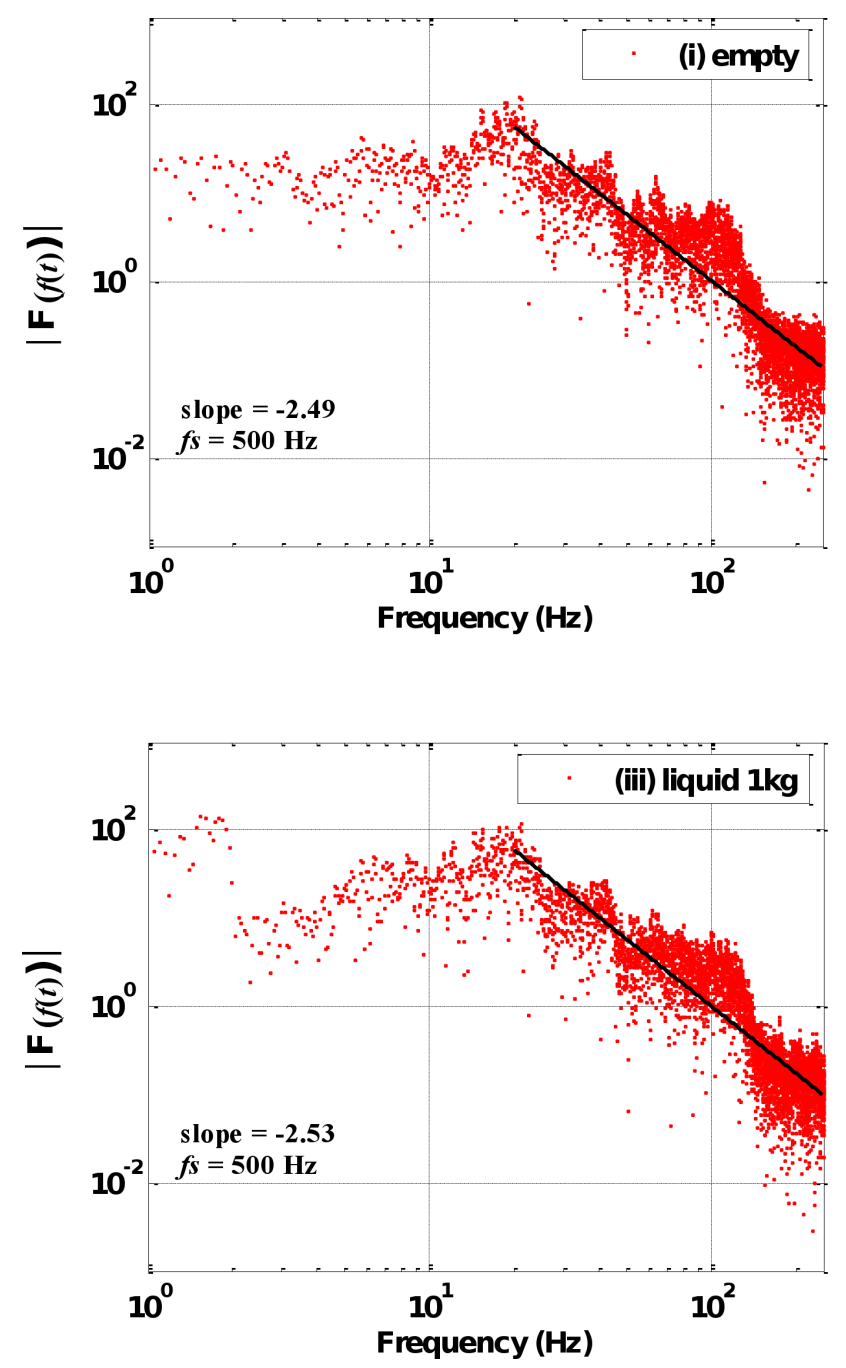

Fig. 11. Spectrum of the $F_{y}$ force for the trapezoidal profile

Figure 10 shows the FFT amplitude of the $F_{x}$ force (case $i$ ) for the trapezoidal profile. A trendline is calculated in a frequency range larger than one decade yielding the slope $m=-2.52$.

Figure 11 shows the FFT amplitude of the $F_{y}$ force (cases $i$ and $\mathrm{iii}$ ) for the trapezoidal profile. The corresponding trendlines (calculated in a frequency range larger than one decade) lead to slopes $m=-2.49$ and $m=-2.53$, for the cases $i$ ) and iii), respectively. The slope values of the force components presented (Figs. 10 and 11) show clearly a fractional order behavior. In general, the forces for the other cases not depict have a spectrum that can be approximated by a trendline in a frequency range greater then one decade. Their trendlines present middle slope values that are difficult to classify in terms of its behavior as fractional or integer order.

Figure 12 shows the FFT amplitude of the $M_{z}$ moment (case ii) for the trapezoidal profile. This spectrum is not so well defined in a large frequency range. Moreover, all moments spectra present identical behavior.

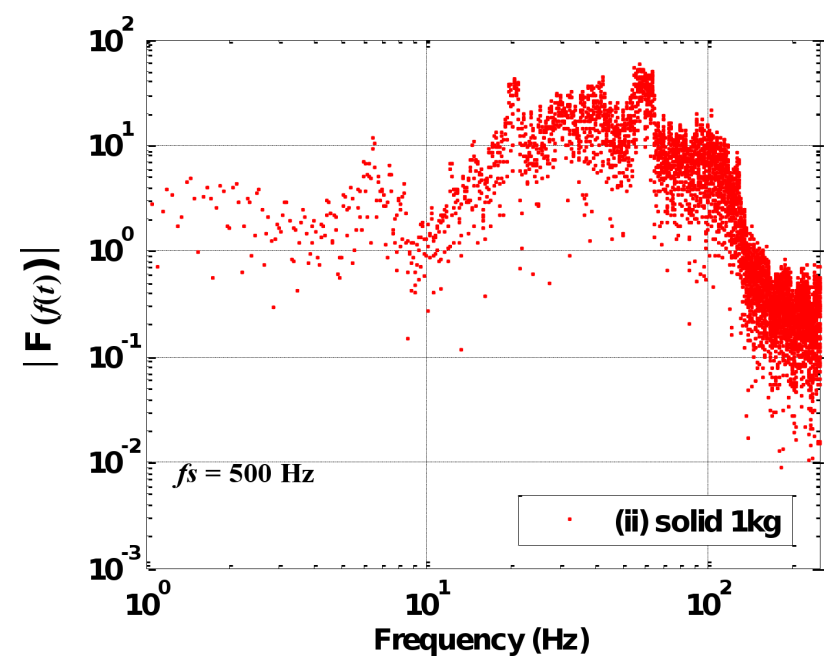

Fig. 12. Spectrum of the $M_{z}$ moment for the trapezoidal profile

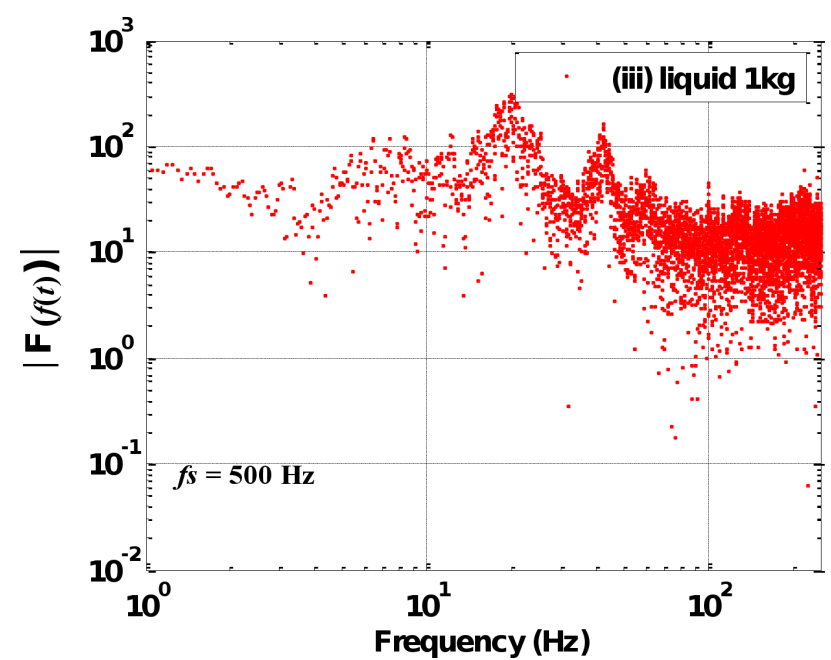

Fig. 13. Acceleration spectrum of the container for the trapezoidal profile

Finally, Fig. 13 depicts the spectrum of the signal captured from the accelerometer 1 located at the container. Like 
the spectrum from the other accelerometer, this spectrum is spread and complicated.

The spectra of the moment and the aceleration signals (Figs. 12-13) are difficult to aproximate by trendlines. Therefore is complicate to define accurately the behavior of signals in terms of integer or fractional dynamics.

The spectra of the captured signals for the trapezoidal profile were studied in terms of their integer versus fractional behavior. The spectra signals for the parabolic profile were also analyzed, but due to space limitations are not presented here. Although the signals in time domain for the parabolic profile present a smoother dynamics, comparing with those of the trapezoidal profile, both spectra reveals identical behavior in terms of integer versus fractional characteristics.

\section{Conclusions}

In this paper an experimental study was conducted to investigate several robot signals during the motion of a liquid container. The amount of slosh depends, among other aspects, on how the container is accelerated. In order to test different acceleration shapes two types of trajectory velocity were used: the trapezoidal and the parabolic profiles. Although the signals in time domain present different dynamics for the two profiles, their spectra reveals identical behavior in terms of integer versus fractional characteristics. The study was conducted in a FC perspective and provides useful information that can assist in the design of a control system to be used in reducing or eliminating the effect of vibrations.

In future work, we plan to pursue several research directions to help us further understand the behavior of the signals. These include the use of a multiwindow algorithm, in order to obtain smoother curves from the scattered spectra.

\section{REFERENCES}

[1] N.C. Singer and W.P. Seering. "Using acausal shaping techniques to reduce robot vibration", Proc. IEEE Int. Conf. on Robotics and Automation 1, CD-ROM (1988).

[2] T. Yoshikawa, K. Hosoda, T. Doi, and H. Murakami. "Quasistatic trajectory tracking control of flexible manipulator by macro-micro manipulator system", Proc. IEEE Int. Conf. on Robotics and Automation 1, 210-215 (1993).

[3] D.P. Magee and W.J. Book, "Filtering micro-manipulator wrist commands to prevent flexible base motion", Proc. American Control Conf. 1, CD-ROM (1995).

[4] D.W Cannon, D.P. Magee, W.J. Book, and J.Y. Lew, "Experimental study on micro/macro manipulator vibration control", Proc. IEEE Int. Conf. on Robotics and Automation 1, CD-ROM (1996).
[5] J.Y. Lew, D.J. Trudnowski, and M.S. Evans, and D.W. Bennett, "Micro-manipulator motion control to suppress macromanipulator structural vibrations", Proc. IEEE Int. Conf. on Robotics and Automation 3, 3116-3120 (1995).

[6] W. Rumold, "Modeling and simulation of vehicles carrying liquid cargo", Multibody Systems Dynamics 5, 351-374 (2001).

[7] M. Grundelius, "Methods for control of liquid slosh", $P h D$ Thesis, Lund Institute of Technology, Lund, 2001.

[8] J.T. Feddema, C.R. Dohrmann, G.G. Parker, R.D. Robinett, V.J. Romero, and D.J. Schmitt, "Control for slosh-free motion of an open container", IEEE Control Systems 17 (1), 29-36 (1997).

[9] J.A.T. Machado, "Analysis and design of fractional-order digital control systems, J. Systems Analysis-Modelling-Simulation 27, 107-122 (1997).

[10] J.A.T. Machado, "A probabilistic interpretation of the fractional-order differentiation", J. Fractional Calculus \& Applied Analysis 6 (1), 73-80 (2003).

[11] I. Podlubny, "Geometrical and physical interpretation of fractional integration and fractional differentiation", J. Fractional Calculus \& Applied Analysis 5 (4), 357-366 (2002).

[12] G.W. Bohannan, "Interpretation of complex permittivity in pure and mixed crystals", www.gl.ciw.edu/ $\sim$ cohen/meetings/ferro2000/proceedings/bohannan.pdf (2000).

[13] G.W. Bohannan, "Analog realization of a fractional control element-revisited mechatronics", ece.usu.edu/foc/cdc02tw/ cdrom/aditional/FOC_Proposal_Bohannan.pdf (2002).

[14] R.S. Barbosa, J.A.T. Machado, and I.M. Ferreira,"Tuning of PID controllers based on Bode's ideal transfer function, Nonlinear Dynamics 38, 305-321(2004).

[15] A. Oustaloup, X. Moreau, and M. Nouillant, "From fractal robustness to non integer approach in vibration insulation: the CRONE suspension", Proc. 36th Conf. on Decision \& Control 1, CD-ROM (1997).

[16] M.F.M. Lima, J.A.T. Machado, and M. Crisóstomo, "Experimental set-up for vibration and impact analysis in robotics", WSEAS Trans. on Systems 5 (4), 569-576 (2005).

[17] M.F.M. Lima, J.A.T. Machado, and M. Crisóstomo, "Fractional order Fourier spectra in robotic manipulators with vibrations", Second IFAC Workshop on Fractional Differentiation and Its Applications 1, CD-ROM (2006).

[18] M.F.M. Lima, J.A.T. Machado, and M. Crisóstomo, "Windowed Fourier transform of experimental robotic signals with fractional behavior", Proc. IEEE Int. Conf. on Computational Cybernetics 1, 21-26 (2006).

[19] M.F.M. Lima, J.A.T. Machado, and M. Crisóstomo, "Fractional dynamics in mechanical manipulation", J. Computational and Nonlinear Dynamics, Transactions ASME 3 (2), CD-ROM (2008).

[20] Scorbot ER VII, "User's manual”, Eshed Robotec, 1996. 